\title{
Hanle and Zeeman effects: from solar to stellar diagnostics
}

\author{
A. López Ariste \\ THEMIS - CNRS UPS 853 \\ C/ Vía Láctea s/n 38205 - La Laguna, Spain \\ email: arturo@themis.iac.es
}

Résumé. We suggest the use of the area asymmetries of the Stokes V profile of a line sensitive to the Zeeman effect to diagnose variatios of the magnetic field along the line of sight in stellar atmospheres. This tool could allow to disentangle the magnetic topology of the observed stellar features in analogy to the solar case : a fibril topology as in plage and netwrok magnetic fields vs. a homogeneous and strong field as in sunspots. We also suggest the use of the Hanle effect as a means to observe weak global dipoles.

Keywords. Polarimetry. Magnetic Fields.

Spectropolarimetry of the solar atmosphere enjoys two advantages over that of other stars. First, the greater photon flux allows higher signal-to-noise ratios at higher spectral resolution and in consequence encourages the study and analysis of subtler polarization effects in spectral lines. Second the spatial resolution of the solar atmosphere allows the observer to distinguish localized features and structures and to propose more detailed models for, among others, the magnetic fields in those regions. Because of those advantages solar spectropolarimetry has explored and taken advantage of subtle polarization signatures that, we suggest, can now be exported to stellar spectropolarimetry. In this contribution I present and propose two of those subtle signatures as tools : the asymmetries of the Zeeman-due Stokes V profile and the Hanle effect.

Under the Zeeman effect, the spectral profile in Stokes V of a magnetically sensitive line shows a characteristic antisymmetric profile with two identical lobes of opposite sign. In the usual case (usual in the observation of solar and stellar photospheres) of a Zeeman splitting smaller than or comparable to the width of the spectral line (a width due to thermal, rotational or other broadening effects) the lobes are fixed in position and its amplitude is proportional to the longitudinal magnetic flux, where by longitudinal we mean the projection of the magnetic vector over the line of sight. This is what is often called the weak-field regime of the Zeeman effect and it is commonly used in Zeeman Magnetic Imaging of stellar photospheres or in many solar magnetographs. The previous description is true as long as the magnetic field is constant over the formation region of the line observed. This may be a good starting approximation, but it is clear that eventually we should consider that the magnetic field can change along the path of the photon through the stellar atmosphere (Westendorp Plaza et al., 2001a, Westendorp Plaza et al. 2001b). The effect of this variability on the Stokes V profile is to make the two lobes asymmetric : they will no longer have the same amplitude and the areas enclosed by them will be different with the result of a net circular polarization when integrated in wavelength (López Ariste, 2002). The observation of such asymmetries in Stokes $\mathrm{V}$ profiles is therefore a potential diagnostic of the variability of the magnetic field in the stellar atmosphere. 
Two kinds of asymmetry appear : the amplitudes of the lobes may be different, and the areas enclosed by the lobes may be different. Of these two kinds it is the last one that carries the diagnostic potential. It is possible to make the amplitudes of the lobes differ by, for example, simple addition of different Stokes profiles with different Doppler shifts arising from different points in the photosphere. On the other hand there is only one way to produce an area asymmetry $\delta A$ : to have simultaneously non-zero gradients of the magnetic $B$ and velocity $v$ fields with opacity $\tau$ along the path of the photon .

$$
\delta A=\frac{\int V d \lambda}{\int\|V\| d \lambda} \propto \frac{d B}{d \tau} \frac{d v}{d \tau}
$$

The addition of symmetric Stokes V profiles, of different amplitudes and at different Doppler shifts will not change the integral over wavelength, it will not introduce any area asymmetry. Thus, the observation of area asymmetry unambiguously point towards gradients of magnetic and velocity fields along the path of the photon. There is a second remarkable aspect of area asymmetries of Stokes V profiles. One can define an area sign by always subtracting, let's say, the red lobe from the blue lobe. Is there a preferred sign for the area asymmetry? In the solar photosphere the answer is yes (GrossmannDoerth et al., 2000). Due to the thermal structure of the solar atmosphere, the expected increase of magnetic fields with depth often results in the red lobe having a larger area than the blue one. This has two immediate implications when translating this diagnostic tool to stellar observations : first it is difficult to erase the area asymmetry from the disk-integrated Stokes V profile if one lobe is larger than the other over large spans of the atmosphere; second it suggests a direct comparison with the solar case. Is the sign the same as in the solar photosphere? Are amplitudes comparable? Is this what the Sun would look like?

Observations of stellar Stokes V profiles have shown the presence of area asymmetry (Petit et al., 2013, Tsvetkova et al., 2013). The interpretation of those will pass through the introduction of gradients of magnetic field. The solar case has produced models of magnetic field topology that can be told apart in terms of the area asymmetry of Stokes $\mathrm{V}$ profiles. As a suggested path towards the interpretation of area asymmetries in stellar Stokes $\mathrm{V}$ profiles we can suggest the following dictionary :

Solar plages, magnetic network: These solar features are characterized by relatively weak fields covering large areas of the photosphere with the same polarity. In stellar observations, one is tempted to associate them with the so-called small-scale magnetic structures. The magnetic topology of those features is best described as fibril : collections of vertical flux tubes where magnetic field concentrates and filling just a fraction of the atmosphere with no or very weak field outside them. In almost every ray path that one can trace through such a topology, the photon will cross several times those high-field

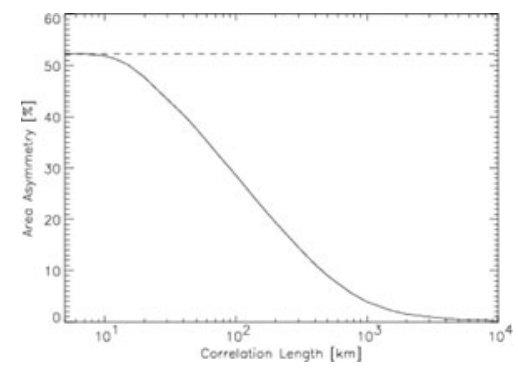

Fig. 1. Relationship between the area asymmetry of the Stokes V profile and the characteristic scales of change of the magnetic field (from Carroll \& Staude, 2005) 
flux tubes. The Stokes V profile will therefore show area asymmetries. If the comparison is correct, we should expect small-scale magnetic fields in stars to show clear area asymmetries.

Sunspots : The magnetic field in sunspots is homogeneous, but their size is relatively small when compared to plages or network fields. Because of this it is tempting to say that they are not observed in most of the stellar observations. The homogeneity of the magnetic field results in a zero or near-zero area asymmetry

Global field: This is the magnetic field of the star as a whole. Although in times of solar maximum its structure may be complex, the global field can be thought of as made of a low-order polar composition with scales of change comparable to the radius of the star. This is referred to as the large scale field in the stellar context. Because of the large scale of these fields, the photon has few opportunities to see gradients and one expects low or zero area asymmetries in the respective Stokes V profiles

The observation of area asymmetries in stars then points to the presence of plage and network fields, with their fibril topologies, as the ones more often seen in stars.

Zeeman effect is sensitive to magnetic fields in the range of hundreds to thousands of G. Although fields of the order of few $\mathrm{G}$ are often mentioned as the result of the measurement of Zeeman effect, it must be stressed that those measurements refer to flux densities which should have been measured in $M x / \mathrm{cm}^{2}$. In a homogeneous field, flux density is equal to intensity and $M x / \mathrm{cm}^{2}$ are $\mathrm{G}$. But in a non homogeneous field the equivalence breaks and it is an abuse of language to use $\mathrm{G}$ as the measurement unit. However distributed, whatever is final flux density, the fields to which our Zeeman effect tools are sensitive are in the range of hundreds to thousands of G. In order to measure weaker field strengths another physical effect is used in solar physics : the Hanle effect.

Hanle effect is a modification of the atomic polarization by the magnetic field (Landi Degl'Innocenti and Landolfi, 2004). By atomic polarization we refer to the presence of quantum coherences among the different atomic levels and/or terms. Since we modify it, it has to be nonzero to start with. The most common source of atomic polarization is illumination by an anisotropic radiation field. Hanle effect is therefore related to lines formed by scattering. The best example is that of solar prominencesCasini et al., 2003 : clouds of cold and dense plasma supported by magnetic fields in the midst of the rarefied and hot corona. Prominence plasma is illuminated by a cone of radiation from the photosphere which induces atomic polarization. Hanle effect modifies it in the presence of magnetic fields of strength such that induces a Zeeman splitting comparable to the natural width of the atomic level. Upon re-emission, the photon is linearly polarized, the amount and plane of polarization depend on the anisotropy of the radiation field and on the magnetic field. But it also depends enormously on the actual atomic structure, what makes Hanle effect always difficult to describe.

Since the polarization is attached to a scattering process, when integrated over a stellar disk the net result is zero. In order to use the Hanle effect in stellar observations there must be some break in that spherical symmetry. The presence of accretion disks or huge prominences may be one reason for that symmetry breaking. Non-spherical stars may also break that symmetry. Here we present another possibility introduced by López Ariste et al. (2011) in which it is the Hanle effect itself that breaks that symmetry when it is due to a dipole global field. Hanle effect diminishes the polarization rate at certain positions of the disk, depending on the field strength but mostly on the geometry of the field respect to the radiation field from the photosphere and its position on the disk. These local modifications of the scattering polarization break the spherical symmetry and result in a net polarization in those lines sensitive to the Hanle effect. The mechanism 

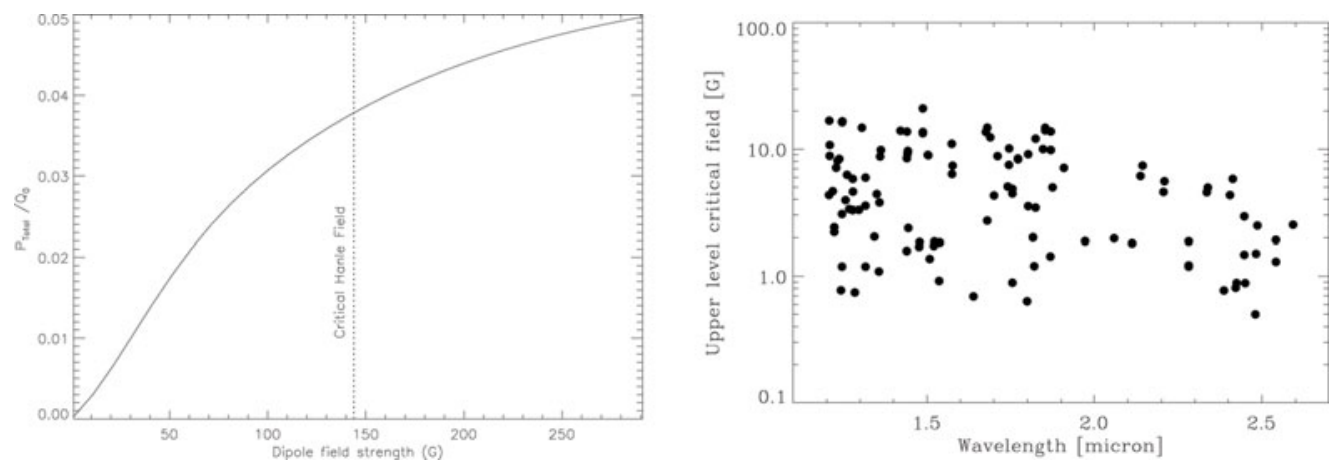

Fig. 2. Left : Variation of expected integrated linear polarization due to the Hanle effect of a dipole as a function of its magnetic strength, for a spectral line with critical Hanle field of around 150G. Right : Compendium of critical Hanle fields for lines in the solar spectrum (from López Ariste et al., 2011)

works for dipoles with strengths well below 100G and though interesting it carries the disadvantage that the actual polarization signals are very weak, perhaps in the order of $10^{-4}$ times the intensity of the continuum.

We have presented two diagnostic tools in use in solar magnetism that can be exported to the stellar case. The first one is the area asymmetry of the Stokes V profile. This asymmetry depends on the simultaneous presence of gradients in the magnetic and velocity fields along the path of each photon. The observation of these asymmetries reveals therefore the presence of non-constant magnetic fields. Sunspots are not expected to show much asymmetries, but the more extended plage and network fields with their fibril field topology do. If the small scale fields observed in stars correspond to these solar magnetic topologies, they should be identifiable through area asymmetries in their Stokes V profiles, even after integration over the stellar disk. The second diagnostic tool is Hanle effect, which is sensitive to weak fields. Hanle effect is associated to scattering processes and therefore cancels out when integrated over the stellar disk unless something breaks the spherical symmetry. One such case is illustrated, as a global dipole field resulting in a net linear polarization which carries the signature of the dipole field.

\section{References}

Carroll, T. A. \& Staude, J. 2005. Astronomische Nachrichten 326, 296-300.

Casini, R., López Ariste, A., Tomczyk, S., \& Lites, B. W. 2003. The Astrophysical Journal Letters 598, L67-L70.

Grossmann-Doerth, U., Schüssler, M., Sigwarth, M., \& Steiner, O. 2000. Astronomy and Astrophysics $357,351-358$.

Landi Degl'Innocenti, E. \& Landolfi, M. 2004. Polarization in Spectral Lines. volume 307. Kluwer Academic Publishers, Dordrecht.

López Ariste, A. 2002. The Astrophysical Journal 564, 379-384.

López Ariste, A., Asensio Ramos, A., \& González Fernández, C. 2011. Astronomy and Astrophysics 527, 120.

Petit, P., Aurière, M., Konstantinova-Antova, R., Morgenthaler, A., Perrin, G., Roudier, T., \& Donati, J. F. 2013, p. 231.

Tsvetkova, S., Petit, P., Aurière, M., Konstantinova-Antova, R., Wade, G. A., Charbonnel, C., Decressin, T., \& Bogdanovski, R. 2013. Astronomy and Astrophysics 556, 43.

Westendorp Plaza, C., del Toro Iniesta, J. C., Ruiz Cobo, B., \& Martínez Pillet, V. 2001a. The Astrophysical Journal 547, 1148-1158.

Westendorp Plaza, C., del Toro Iniesta, J. C., Ruiz Cobo, B., Martínez Pillet, V., Lites, B. W., \& Skumanich, A. 2001b. The Astrophysical Journal 547, 1130-1147. 